\title{
Klavikulafrakturen im Wachstumsalter
}

Wolfram Korsch

\section{Entstehung, Entwicklung, Form, anatomische Besonderheiten}

Die Klavikula entsteht, wie die Skelettelemente des Schädeldaches und nahezu alle Knochen des Gesichtsschädels, unmittelbar aus dem Mesenchym (desmale Ossifikation) und nicht über eine knorpelige Voranlage wie die meisten anderen Extremitätenknochen (chondrale Ossifikation). Mit der Ossifikation des Schlüsselbeines beginnt in der 7. Embryonalwoche die Entstehung des Skeletts [1].

Am medialen, dem Brustbein zugewandten Ende bildet sich um das 18. Lebensjahr ein Knochenkern, der häufig erst zwischen dem 20. und 24. Lebensjahr verknöchert. Das Gelenk zwischen Schlüsselbein und Brustbein stellt den einzigen gelenkigen Kontakt zwischen dem Schultergürtel und dem Rumpf dar. Nach lateral hin bildet das Schlüsselbein mit dem Akromion das Schultereckgelenk [2].

Der leicht S-förmig gebogene Knochen ist medial etwas verdickt und nach lateral hin abgeflacht und verbreitert. Am Unterrand des lateralen Drittels finden sich die Verankerungsstellen der Bandverbindungen zum Processus coracoideus des Schulterblattes. Im Bereich der Schaftmitte unterkreuzen $A$. und V. subclavia, medial befinden sich der Venenwinkel und die Mediastinalgefäße. Die 1. Rippe, die am Übergang zum medialen Drittel kreuzt, kann als Hypomochlion wirken.

Zur Verschiebung von Fragmenten des Schlüsselbeines können zum einen der M. sternocleidomastoideus (Zug des medialen Fragmentes nach kranial), zum anderen die $\mathrm{Mm}$. pectoralis und subclavius (Verkürzung) führen [3].

Besondere Bedeutung wird dem äußerst dicken Periostschlauch der Klavikula im Kinder- und Adoleszentenalter beigemessen. Dieser stellt einen gewissen Schutz vor Verschiebungen dar und beeinflusst die Verletzungsmorphologie an der lateralen Klavikula im Vergleich zum Erwachsenen (s.u.) [3].

\section{Wachstumsbeeinflussung, Korrekturmöglichkeiten}

Wachstumsstörungen sind i.d. R. nicht zu erwarten. Die „Spontankorrektur“ von frakturbedingten Fehlstellungen (Seit-zu-Seit-Verschiebungen und Verkürzungen) können, insbesondere bei Kindern unterhalb des 10. Lebensjahres, mit in die Therapieentscheidung einbezogen werden [3-5].

\section{Frakturhäufigkeit, -entstehung, -lokalisation, -klassifikation}

Frakturen des Schlüsselbeines gehören zu den häufigen Frakturen. In der Literatur variieren die Angaben zwischen 5 und 20\% der Frakturen im Kindesalter [3, 5-7].

Als Entstehungsmechanismus wird überwiegend ein Sturz auf die Schulter und eine dadurch entstehende axiale Stauchung der Klavikula beschrieben. Neben Kontaktsportarten sind Stürze beim Skifahren und/oder Radfahren geeignete Frakturursachen. Daneben ist auch eine direkte Krafteinwirkung auf das Schlüsselbein möglich. Seltener ist dies durch einen Schlag verursacht, als vielmehr durch Rückhaltesysteme im Verkehr [3].

Mit 3-8 Klavikulafrakturen pro 1000 Geburten stellt sie die häufigste geburtstraumatische Verletzung dar $[3,4]$.

Bei Säuglingen und noch immobilen Kleinkindern sollte bei inadäquatem Unfallmechanismus und ggf. weiteren Auffälligkeiten wie multiplen Hämatomen auch an eine Kindesmisshandlung gedacht werden $[3,8]$.

Die gängigste Klassifikation der Schlüsselbeinbrüche ist die nach Allman [9]. Die Klavikula wird in 3 Segmente eingeteilt ( $\bullet$ Abb. 1). Typ I stellt den Schaft dar, Typ II den lateralen Bereich jenseits der korakoklavikulären Bänder, Typ III den Abschnitt medial des kostoklavikulären Bandes. Die Typen können noch weiter unterteilt werden nach undisloziert, disloziert und mehrfragmentär ( $\triangleright$ Tab. 1).

Den überwiegenden Anteil der Frakturen stellen mit über 90\% die Typ-I-Frakturen, etwa die Hälfte als Grünholzfrakturen $[3,4]$. 
Die medialen Typ-III-Verletzungen sind äußerst selten und kommen in 3 Erscheinungsformen vor: zum einen als Verletzung der medialen Wachstumsfuge, wiederum unterteilt nach Salter/Harris [10], zum anderen echte Schaftbrüche zwischen medialer Fuge und dem Ansatz des M. sternocleidomastoideus. Mediale Luxationen dürften eine Rarität darstellen oder eher missinterpretierten Fugenverletzungen entsprechen [3]. Wichtiger ist die Dislokationsrichtung des Schaftanteils, wobei die seltenere posteriore die bedrohlichere ist.

Die Typ-II-Verletzungen nach Allman im Kindesalter werden in Anlehnung an die Akromioklavikulargelenkverletzungen (AC-Gelenkverletzungen) des Erwachsenen auch nach Dameron und Rockwood eingeteilt [11]. Aufgrund des äußerst kräftigen Periostschlauches kommt es i.d.R. zu einer Durchspießung des Schlauches durch das mediale Schaftfragment, wobei der Schlauch mit den straffen korakoklavikulären Bändern am lateralen Fragment stehen bleibt. Die Einteilung erfolgt nach der Dislokationsrichtung des Schaftfragmentes ( $\mathbf{A} \mathbf{A b} \mathbf{b} . \mathbf{2}$ ). Der Periostschlauch kann sich durch Förderung der periostalen Ossifikation zu einer „Neoklavikula“ bilden (

Bei Adoleszenten, bei denen der Periostschlauch nicht mehr diese Ausprägung aufweist, entstehen neben „echten“ AC-Gelenkverletzungen, die nach Rockwood zu klassifizieren sind [3, 12], auch laterale Klavikulafrakturen. Da bei diesen Verletzungen die Relation zur Lage der korakoklavikulären Bänder entscheidend ist, werden sie wie beim Erwachsenen nach Jäger und Breitner klassifiziert ( Abb. 3; Tab. 3) [13].

\section{Diagnostik}

Führend in der Diagnostik ist zu allererst das klinische Bild mit Schmerzen über dem Schlüsselbein, einhergehend mit einer Bewegungseinschränkung der Schulter der betroffenen Seite. Je jünger das Kind, umso unspezifischer sind die Schmerzangaben; häufig fällt lediglich eine „Pseudoparalyse“ des Armes auf. Da eine Schlüsselbeinfraktur wesentlich häufiger auftritt als eine Oberarmfraktur sollte in erster Linie die Klavikula abgeklärt werden.

Den Grundprinzipien der Diagnostik beim Kind folgend ist eine ausgedehnte palpatorische Funktionsprüfung des Schultergürtels zu vermeiden. Ein Zugewinn an Informationen hinsichtlich der Therapieentscheidung ist nicht zu erwarten, stattdessen wird dem Kind nur zusätzlicher Schmerz zugefügt und es wird eher verunsichert [14]. Der neurovaskuläre Status ist aber ebenso zu fordern wie die Dokumentation von Hauteinziehungen über dem Knochen oder gar Hautdurchspießungen.

In der Hand des Erfahrenen ist bei klinischem Verdacht, aber nicht vorhandener Fehlstellung, die Sonografie ein sicheres Verfahren zur Primärdiagnostik. Aufgrund des

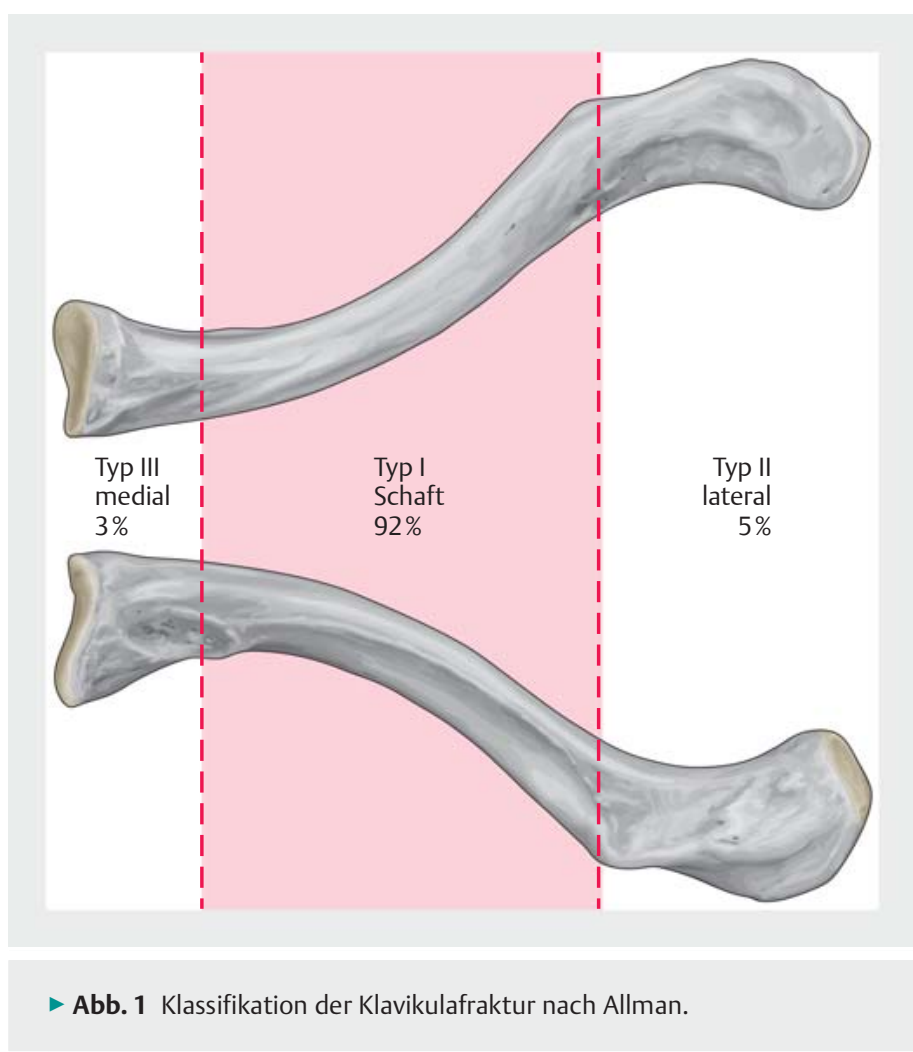

- Tab. 1 Frakturklassifikation nach Allman.

\begin{tabular}{|l|l|}
\hline Typ I & Frakturen im mittleren Drittel („Schaft“) \\
\hline Typ II & Frakturen lateral der korakoklavikulären Bänder \\
\hline Typ III & Frakturen medial des kostoklavikulären Bandes \\
\hline Subtypen & $\begin{array}{l}\text { A: undisloziert } \\
\text { B: disloziert } \\
\text { C: mehrfragmentär }\end{array}$ \\
\hline
\end{tabular}

schmalen Weichteilmantels ist der Knochen gut beurteilbar [15].

Die konventionelle Röntgendiagnostik stellt aber weiterhin den Goldstandard in der Bildgebung am Schlüsselbein dar. Neben der a.-p. Projektion kommt i.d. R. noch eine Tangentialaufnahme zum Einsatz, um eine Dislokation in der sagittalen Richtung zu erkennen. Spezialeinstellungen bleiben besonderen Fragestellungen, insbesondere an der lateralen Klavikula, vorbehalten.

Aufgrund der ausgeprägten Überlagerungen am klavikosternalen Übergang kann hier die CT für Klarheit sorgen, wobei wegen der hohen Strahlenbelastung auch über eine MRT-Bildgebung diskutiert werden sollte. 

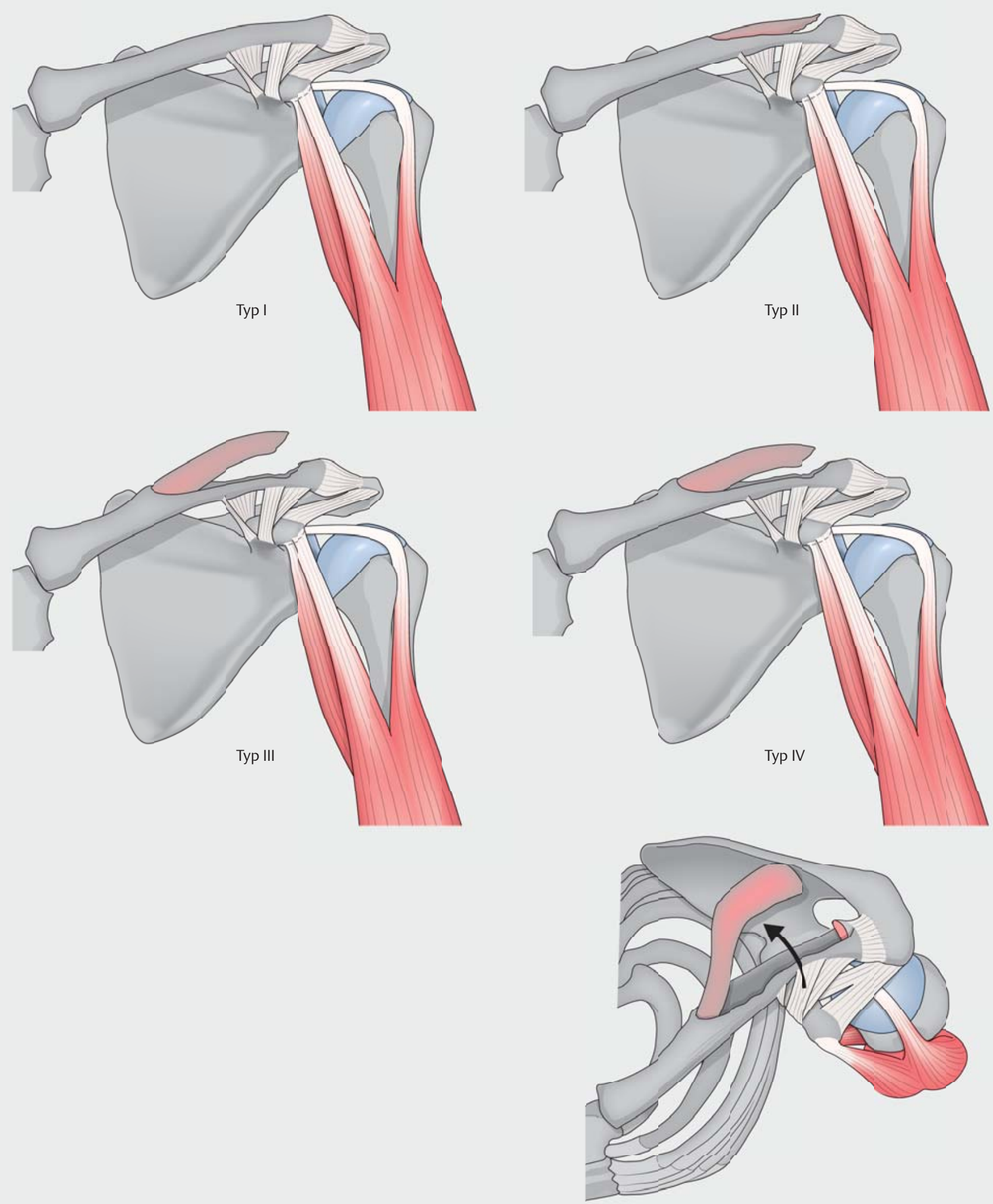

- Abb. 2 a Klassifikation der lateralen Frakturen nach Dameron und Rockwood. Quelle: Leiblein M, Voth M, Marzi I. Frakturen und Gelenkverletzungen an der oberen Extremität beim Kind, Teil 1. Orthopädie und Unfallchirurgie up2date 2018; 13: 275-296. 

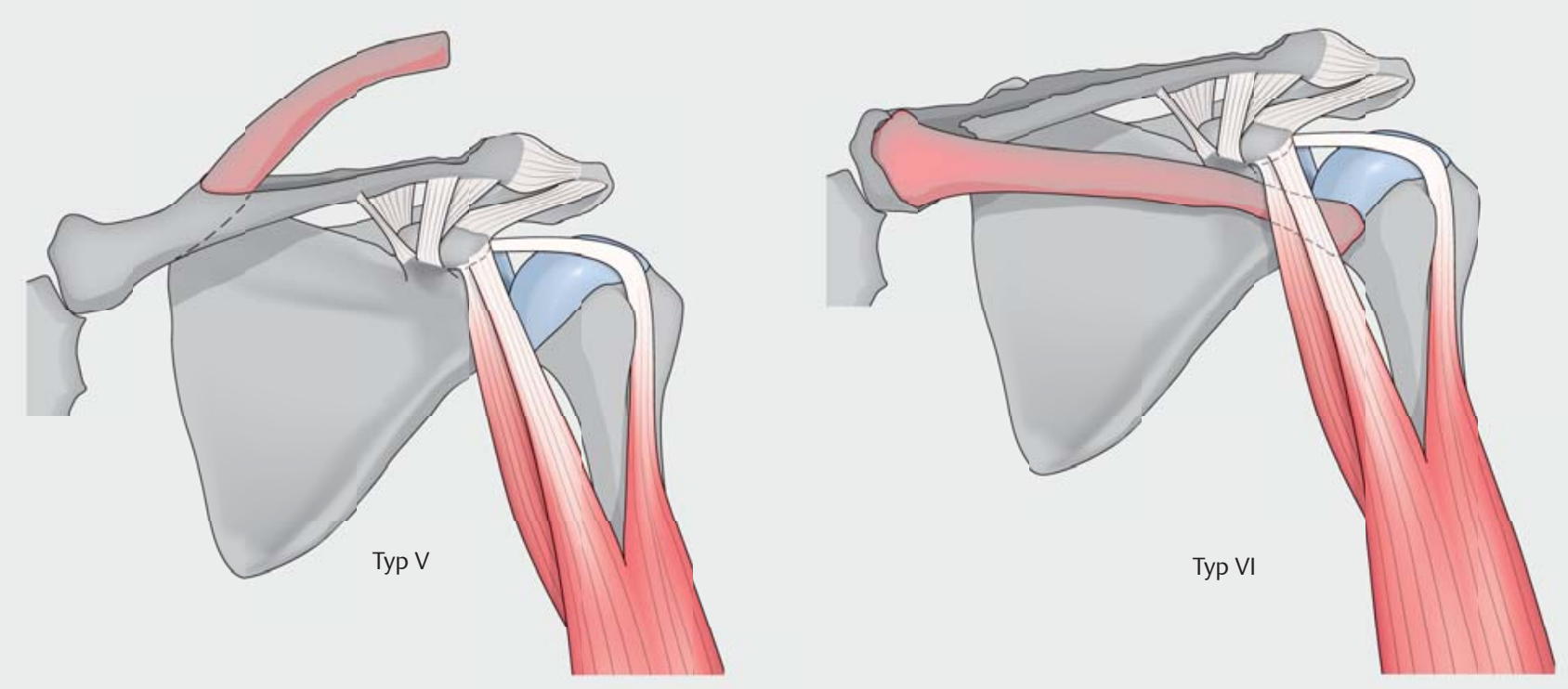

- Abb. 2 b Klassifikation der lateralen Frakturen nach Dameron und Rockwood. Quelle: Leiblein M, Voth M, Marzi I. Frakturen und Gelenkverletzungen an der oberen Extremität beim Kind, Teil 1. Orthopädie und Unfallchirurgie up2date 2018; 13: 275-296.

- Tab. 2 Frakturklassifikation der lateralen Klavikulafraktur nach Dameron und Rockwood [3, 11].

\begin{tabular}{|c|l|}
\hline Typ I & Dehnung des Lig. acromioclaviculare oder Fissur der lateralen Klavikula ohne Läsion des Periostschlauches, keine Dislokation sichtbar \\
\hline Typ II & $\begin{array}{l}\text { partielle Perforation und Riss des Periostschlauches, geringe Instabilität mit gering erweitertem Frakturspalt ohne wesentliche Vergröße- } \\
\text { rung der akromioklavikulären Distanz }\end{array}$ \\
\hline Typ III & $\begin{array}{l}\text { erweiterter Riss des Periostschlauches mit deutlicher Instabilität und Hochstand des medialen Fragmentes; korakoklavikuläre Distanz auf } \\
25-100 \% \text { erweitert }\end{array}$ \\
\hline Typ IV & $\begin{array}{l}\text { wie Typ III mit dorsaler Dislokation des medialen Fragmentes; ggf. Perforation des Fragmentes in den M. trapezius; im a.-p. Strahlengang } \\
\text { unterschätzbar }\end{array}$ \\
\hline Typ V & $\begin{array}{l}\text { komplette Spaltung des Periostschlauches, Dislokation des medialen Fragmentes nach proximal subkutan; korakoklavikuläre Distanz um } \\
\text { über 100\% erweitert }\end{array}$ \\
\hline Typ VI & nach distal disloziertes mediales Fragment, mit Verhakung unter Processus coracoideus \\
\hline
\end{tabular}

- Tab. 3 Frakturklassifikation der lateralen Klavikulafraktur des Adoleszenten nach Jäger und Breitner [3, 9].

\begin{tabular}{|l|l|l|}
\hline Typ & Frakturlokalisation & begleitende Bandverletzung \\
\hline Typ I & Fraktur lateral der CC-Bänder & ggf. Lig. acromioclaviculare \\
\hline Typ II a & Fraktur im Ansatzbereich der CC-Bänder & Ruptur Pars conoidea des Lig. coracoclaviculare \\
\hline Typ IIb & Fraktur im Ansatzbereich der CC-Bänder & Ruptur Pars trapezoidea des Lig. coracoclaviculare \\
\hline Typ III & Fraktur medial der CC-Bänder & keine \\
\hline Typ IV & Aushülsverletzung der lateralen Klavikula aus Periostschlauch & keine \\
\hline CC-Bänder: korakoklavikuläre Bänder & \\
\hline
\end{tabular}




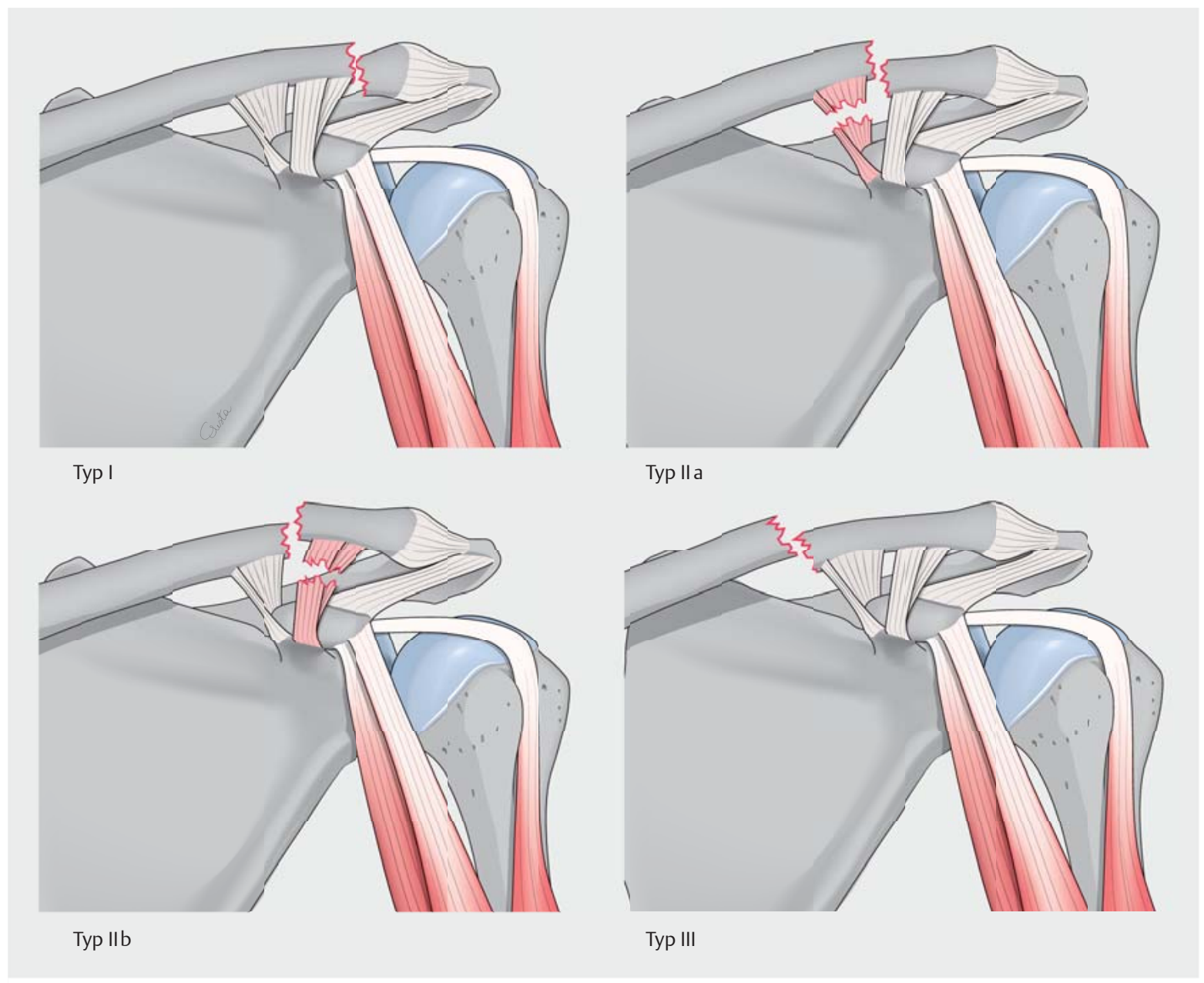

- Abb. 3 Klassifikation der lateralen Fraktur des Adoleszenten nach Jäger und Breitner.

\section{Therapie}

\section{Grundsätzliche Überlegungen zur Therapie im Wachstumsalter}

Die Grundziele der Therapie von Verletzungen im Wachstumsalter sind vor allem durch folgende Punkte gekennzeichnet [14]:

- rasche Schmerzfreiheit

- keine zusätzliche Traumatisierung

- rasche Wiederherstellung der Spiel- und Sportfähigkeit

- effiziente Diagnostik und Behandlung

- Vermeidung von Komplikationen (Infekte, Gefäß-Nerven-Verletzungen, Therapiewechsel etc.)

Neben der grundsätzlichen Unterscheidung zwischen konservativen und operativen Maßnahmen wird von manchen Autoren eine zusätzliche Differenzierung zwischen konservativer Therapie ohne und mit Anästhesie gefordert [14].

\section{Konservative Therapie}

Bei Kindern unter 2 Jahren ist eine Heilung der Fraktur in gut 2 Wochen ohne spezielle Therapie zu erwarten. Primäres Ziel ist auch hier die ausreichende Analgesie, die durch eine Ruhigstellung in einer Armschlinge erreicht werden kann. Bei unruhigen Säuglingen ist ggf. ein Anheften des Ärmels des Stramplers am Brustteil zweckmäßig.

Bei älteren Kindern bieten sich für die Ruhigstellung im Rahmen der konservativen Therapie, die die Domäne der Therapie darstellt, verschiedene Techniken an. Der nach wie vor zur Anwendung kommende Rucksackverband stellt in unseren Augen keine adäquate Methode mehr dar. Der durch ein regelmäßiges „Nachziehen“ des Verbandes erhoffte Repositionserfolg stellt sich meist nicht ein, vielmehr überwiegen die Komplikationen wie Hautverletzungen, Kribbeldysästhesien der Arme oder Störungen der Armdurchblutung. Einziger Vorteil gegenüber einer kindgerechteren Armschlinge ist eine verbleibende Funktion der Hand. [3, 14] 

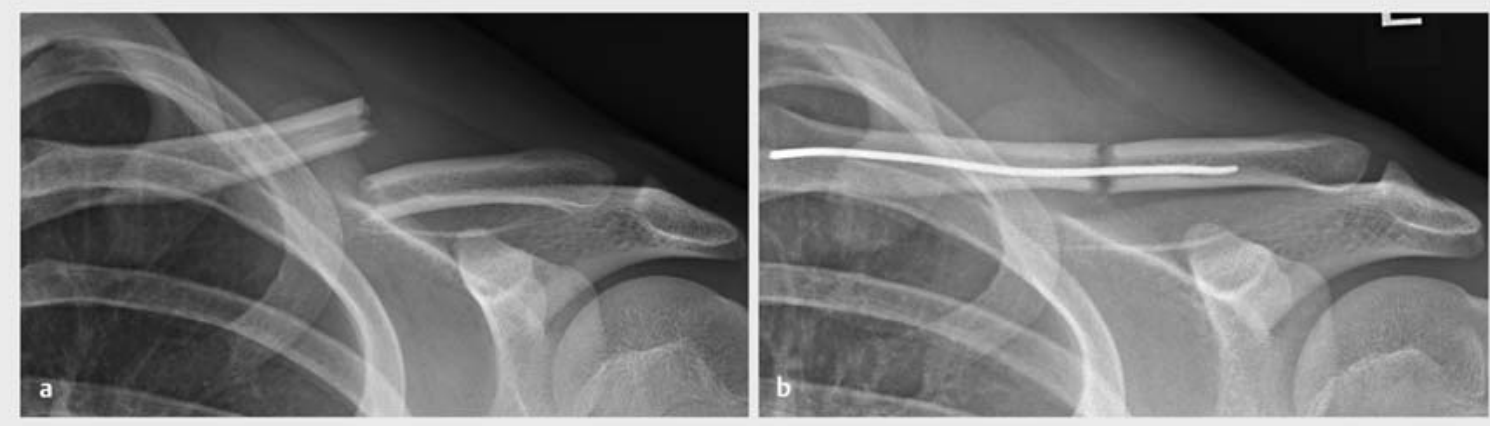

- Abb. 4 Schaftfraktur eines 16-jährigen Jungen: a Unfallbild mit Dislokation um fast doppelte Schaftbreite. b Postoperative Situation nach offener Reposition und intramedullärer Schienung. Quelle: Donau-Ries Kliniken, Donauwörth.

Im Rahmen der konservativen Behandlung kommt der klinischen Verlaufskontrolle eine entscheidende Bedeutung zu. Bei dem direkt subkutan liegenden Knochen kann zumindest der Verdacht auf eine sekundäre Stellungsänderung durch vorsichtige Palpation gestellt werden. Vor allem bei Kindern über 10 Jahren sollte aufgrund der eingeschränkten Spontankorrektur eine sekundäre Fehlstellung (insbesondere Verkürzung) ausgeschlossen werden. Bei klinischem Verdacht sollte daher die Indikation zur radiologischen Stellungskontrolle innerhalb der ersten beiden Wochen nach Trauma nicht zu streng gestellt werden.

Die Ruhigstellungsdauer richtet sich nach der Schmerzhaftigkeit der Frakturregion. In der Regel kann bereits nach 3 Wochen der betroffene Arm wieder einigermaßen spontan bewegt werden. Zu diesem Zeitpunkt kann die Ruhigstellung beendet werden und dem Patienten die Eigenmobilisation bis zur Schmerzgrenze unterhalb der Horizontalen gestattet werden. Sobald der entstehende Frakturkallus auf direkten Druck nicht mehr schmerzhaft ist kann die Fraktur als konsolidiert bewertet werden. Bei erreichter freier Schulterbeweglichkeit ist die Wiederaufnahme der sportlichen Aktivität zu erlauben [4, 5].

\section{Operative Therapie}

Neben offenen Frakturen stellen grob dislozierte Brüche, ggf. mit drohender Hautperforation, und Brüche mit begleitenden Gefäß-Nerven-Schäden eine klare Indikation zur operativen Versorgung dar [3].

Als relative OP-Indikationen gelten: „Klavikulafrakturen beim polytraumatisierten Patienten, beidseitige Frakturen, Mehrfachverletzungen i.S. einer Floating Shoulder, relevante Verkürzung bei Kindern über 10 Jahren, Pseudarthrosen, höhergradige Verletzungen der lateralen Klavikula (laterale Frakturen und AC-Gelenkverletzungen) und nach zentral dislozierte mediale Frakturen mit Kompromittierung der mediastinalen Strukturen“ [3].

\section{Allman-I-Frakturen (Schaft)}

Seit-zu-Seit-Verschiebungen um mehr als Schaftbreite können zur nachfolgenden Verkürzungsfehlstellung führen. Insbesondere die Definition „relevante Verkürzung“ unterliegt einer kontroversen Diskussion. Eine direkte Übernahme der Empfehlungen aus der Traumatologie des Erwachsenen wird als zulässig angesehen aufgrund der insgesamt niedrigen Zahl an Fällen [3, 7]. Bei über 10-Jährigen sollte daher ab einer Verkürzung von $1,5 \mathrm{~cm}$ eine anatomische Längenwiederherstellung angestrebt werden.

Da es sich i.d. R. um einfache Querfrakturen ( $\mathbf{A b b}$. 4) handelt, ist die Methode der Wahl zur Stabilisierung die intramedulläre Schienung mit einem elastisch stabilen Nagel. In Rückenlage wird im Bereich des medialen Knochenendes über eine kurze Hautinzision die Kortikalis unter Bildverstärkerkontrolle (BV-Kontrolle) eröffnet und der elastische Nagel in den Markraum eingebracht. Die übliche Nagelstärke liegt bei 2,0 mm. Die Reposition sollte, wenn möglich, geschlossen erfolgen, wobei Repositionshindernisse über eine kurze Inzision über der Fraktur beseitigt werden können. Nach dem Überbrücken der Frakturzone sollte der Nagel ausreichend weit ins laterale Fragment vorgeschoben werden ( $\bullet$ Abb. 4).

Bei langstreckigen Bruchformen oder Zwischenfragmenten ist eine Plattenosteosynthese das Verfahren der Wahl. Zur Auswahl stehen hier neben den klassischen Drittelrohr- und 3,5-mm-Platten zunehmend auch Systeme der Dimension 2,7 mm zur Verfügung. Diese haben den Vorteil, nicht zu stark aufzutragen.

Bei Nachrepositionen mit sicher offenem Zugang kann ebenfalls eine Plattenosteosynthese zur Vermeidung des 2. Zuganges medial geplant werden ( $\bullet$ Abb. 5).

Bei der offenen Zugangswahl sollte vor allem bei Mädchen auf kosmetische Aspekte geachtet werden. Ein modifizierter „Säbelhiebzugang“ kann später unter BH-Trägern verborgen werden ( $\triangleright$ Abb. 6). 

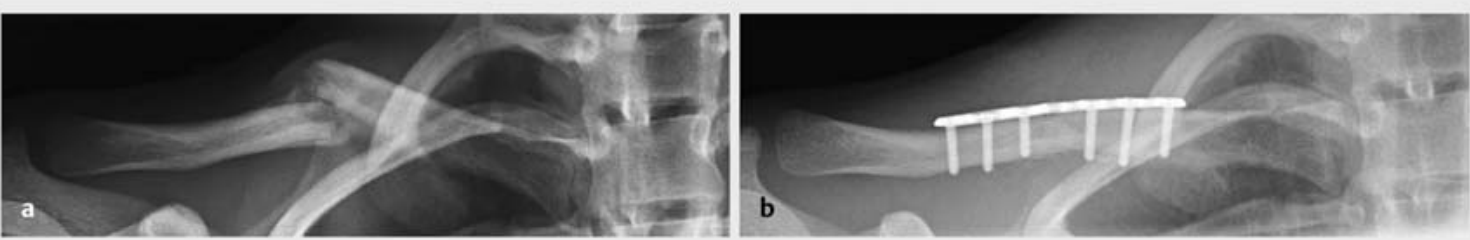

- Abb. 5 a In Fehlstellung verheilte Schaftfraktur mit Verkürzung um ca. 1,5 cm und Vertikalversatz um Schaftbreite. b Postoperative Kontrolle nach offener Korrektur und Plattenosteosynthese. Quelle: Donau-Ries Kliniken, Donauwörth.
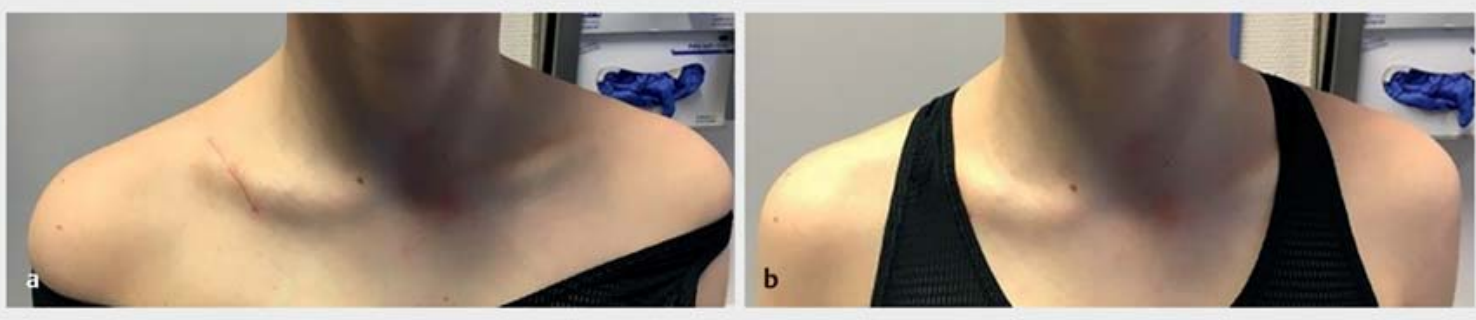

- Abb. 6 Kosmetisches Ergebnis aus \ Abb. 5. Quelle: Donau-Ries Kliniken, Donauwörth.

Allman-II-Frakturen (laterale Klavikula)

Bei diesen Verletzungen stellen bei Kindern unter 10-12 Jahren die Typen IV, V und VI nach Dameron und Rockwood eine OP-Indikation dar. Aufgrund der Dislokation der knöchernen Klavikula aus dem kräftigen Periostschlauch kann es durch den starken Verknöcherungsreiz der Knochenhaut zur Ausbildung einer „Neoklavikula“ neben dem eigentlichen Knochen kommen [3]. Die entstehende „Doppelklavikula“ kann, insbesondere bei Dorsaldislokation des Schaftes, im M. trapezius zur Irritationen führen.

Nach offener Reposition ist eine Naht des Periostschlauches i. d. R. ausreichend zur Retention $[3,5,16]$. Ein Überbrücken des AC-Gelenkes mit Kirschner-Drähten sollte vermieden werden.

Bei Adoleszenten tritt die Stabilität des Periostschlauches in den Hintergrund und die Stabilität wird anhand der Jäger-Breitner-Klassifikation des Erwachsenen abgeschätzt. Die OP-Indikation stellt sich nach der Dislokation. Als Stabilisationsmethoden stehen neben der Zuggurtung und Plattenosteosynthese die Fadenankerrefixation gegen das Korakoid zur Verfügung ( $\bullet$ Abb. 7).

Reine AC-Gelenkverletzungen unterliegen den gleichen OP-Indikationen und Versorgungsstrategien wie beim Erwachsenen [3].
Allman-III-Frakturen (mediale Klavikula)

Die Indikation zur operativen Versorgung am medialen Ende des Schlüsselbeines ergibt sich vor allem bei nach posterior disloziertem Schaftanteil. Diese Verletzungen können zu einer bedrohlichen Kompromittierung der zentralen großen Venen führen und stellen in dieser Situation einen Notfall dar.

Die Reposition erfolgt in Rückenlage, wenn möglich geschlossen. Bei fehlender Stabilität im Anschluss an die Reposition werden zur Refixation Periostnähte oder transossäre Faden-Cerclagen empfohlen [3].

\section{Nachbehandlung}

Im Rahmen der konservativen Therapie sollte die Dauer der Ruhigstellung vor allem klinisch bestimmt werden. Sofern die oben angegebenen Empfehlungen zur radiologischen Stellungskontrolle beachtet werden, kann die Ruhigstellung bei schmerzfreiem Kallus beendet werden. - Tab. 4 gibt eine grobe Richtlinie über die Zeitdauer unter Berücksichtigung des Patientenalters.

Nach Freigabe sollte der Mobilisationsgrad dem Patienten selbst überlassen werden. Physiotherapie ist nur bei anhaltender Funktionseinschränkung nötig [3,14].

Nach osteosynthetischer Frakturversorgung ist je nach Verfahren i.d.R. keine ergänzende Ruhigstellung erfor- 

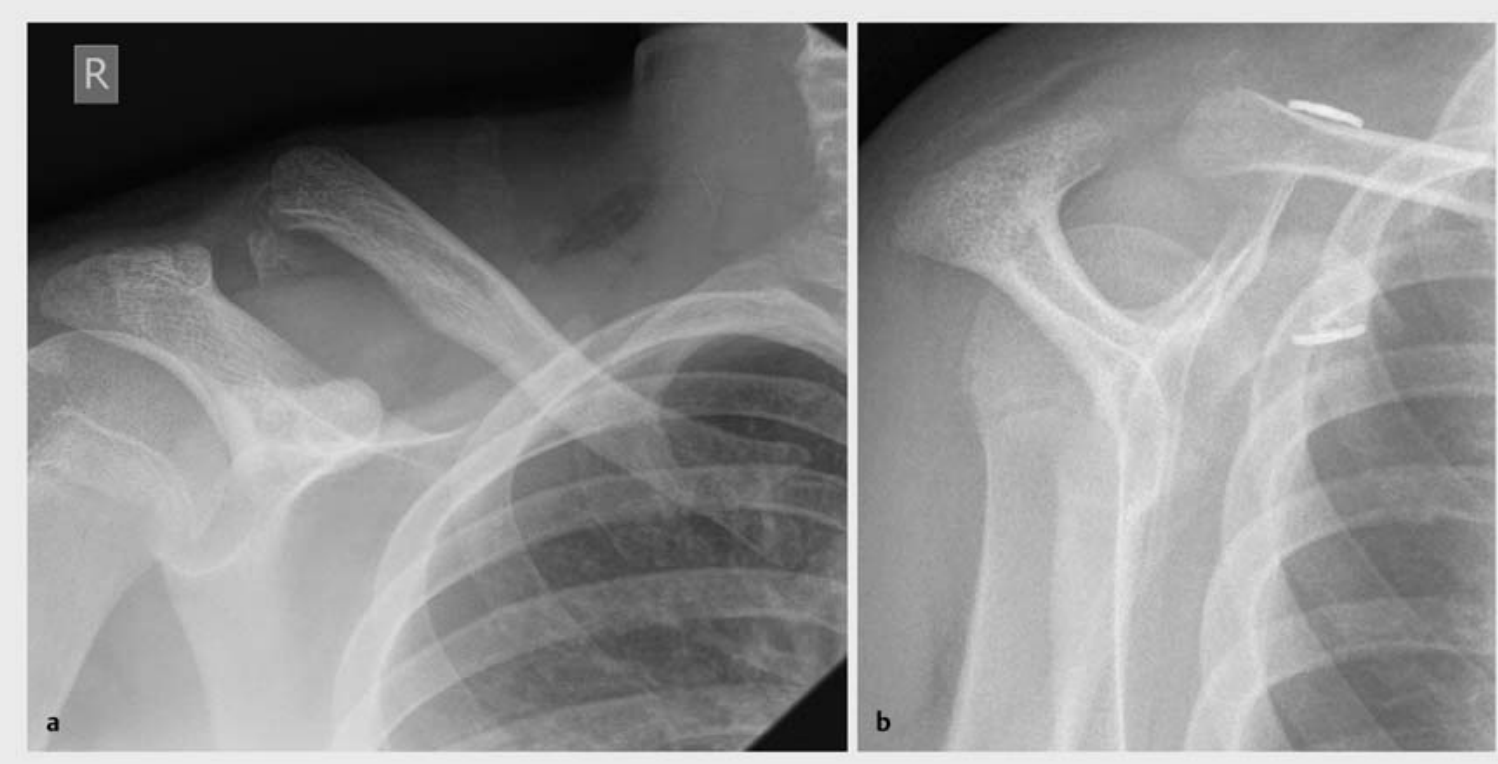

Abb. 7 a Laterale Klavikulafraktur eines 14-jährigen Jungen (Typ IV nach Dameron und Rockwood). b Nach offener Reposition und Fadenankerstabilisation + Periostnaht. Quelle: Donau-Ries Kliniken, Donauwörth.

- Tab. 4 Ruhigstellungs- und Konsolidationszeiträume [3].

\begin{tabular}{|l|l|l|}
\hline Alter & $\begin{array}{l}\text { Ruhigstellungs- } \\
\text { dauer (Wochen) }\end{array}$ & $\begin{array}{l}\text { Konsolidierung } \\
\text { (Wochen) }\end{array}$ \\
\hline < 5 Jahre & 2 & 2 \\
\hline 5-10 Jahre & 3 & 3 \\
\hline > 10 Jahre & 3 & 4 \\
\hline Adoleszente & 3 & $4-6$ \\
\hline
\end{tabular}

derlich. Eine Armschlinge kann in den ersten Tagen zur Analgesie dienen.

Die Selbstmobilisation ist auch hier zu propagieren.

Die Wiederaufnahme der sportlichen Aktivität kann bei freier Beweglichkeit erlaubt werden [4].

Der Zeitpunkt der Metallentfernung ist abhängig vom implantierten Material. Kirschner-Drähte sollten nach 4-6 Wochen entfernt werden. Für elastische Marknägel werden 3 Monate, für Platten ein halbes Jahr empfohlen [3].

\section{Zusammenfassung}

Die Schlüsselbeinfraktur im Kindesalter ist i.d. R. konservativ zu behandeln. Neben den Schaftfrakturen stellen vor allem die Verletzungen im Bereich der lateralen Klavikula eine eigene Entität dar. Zur Ruhigstellung wird eine
Armschlinge empfohlen, da der klassische Rucksackverband durch direkten Druck auf die Fraktur eher mehr Schmerzen verursacht.

Im Falle einer notwendigen operativen Stabilisation der Fraktur ist im Schaftbereich die elastisch stabile Markraumschienung Methode der Wahl. Bei lateralen Frakturen reicht vor allem bei jüngeren Kindern häufig die Naht des Periostschlauches aus, alternativ kommen Zuggurtungen zum Einsatz.

Bei erreichtem schmerzfreien Frakturkallus ist die Selbstmobilisation der routinemäßigen Physiotherapie vorzuziehen. Bei freiem Bewegungsumfang kann die Sportfreigabe erfolgen.

\section{Interessenkonflikt}

Die Autoren geben an, dass kein Interessenkonflikt besteht.

\section{Autorinnen/Autoren}

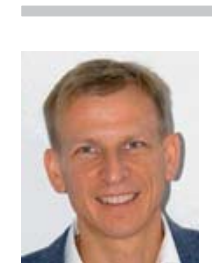

\section{Wolfram Korsch}

Dr. med., Leiter Sektion Unfallchirurgie, Klinik für Orthopädie, Unfallchirurgie, Wirbelsäulentherapie, Donau-Ries Klinik Donauwörth (Klinikdirektor: Prof. Dr. A. T. Wild) 


\section{Korrespondenzadresse}

\section{Dr. med. Wolfram Korsch}

Klinik für Orthopädie, Unfallchirurgie, Wirbelsäulentherapie Donau-Ries Klinik Donauwörth

Neudegger Allee 6

86609 Donauwörth

w.korsch@donkliniken.de

Literatur

[1] Moore KL. Embryologie. 3. Aufl. Stuttgart: Schattauer; 1990

[2] Frick H, Leonhardt H, Starck D. Allgemeine Anatomie - Spezielle Anatomie I. 3. Aufl. Stuttgart: Thieme; 1987

[3] Seif El Nasr M, von Essen H, Teichmann K. Klavikulafrakturen in der Kindertraumatologie. Unfallchirurg 2011; 114: 300-310

[4] von Laer L, Kraus R, Linhart W. Frakturen und Luxationen im Wachstumsalter. 5. Aufl. Stuttgart: Thieme; 2007

[5] Marzi I. Kindertraumatologie. Darmstadt: Steinkopff; 2006

[6] Rehn J. Unfallverletzungen bei Kindern. Heidelberg: Springer; 1974

[7] Trompetter R, Seekamp A. Klavikulafrakturen. Unfallchirurg 2008; 11: 27-39

[8] Stöver B. Bildgebende Diagnostik der Kindesmisshandlung. Radiologe 2007; 47: 1037-1049
[9] Allman FL. Fractures and ligamentous injuries of the clavicle and its articulations. J Bone Joint Surg Am 1967; 49: 774-784

[10] Salter RB, Harris WR. Injuries involving the epiphyseal plate. J Bone Joint Surg Am 1963; 45: 587-622

[11] Dameron TB, Rockwood CA. Fractures and Dislocation of the Shoulder-Clavicle Fractures. In: Rockwood CA, Wilkins KE, eds. Fractures in Children. Philadelphia: Lippincott-Raven; 1984

[12] Rockwood CA jr. Injuries to the acromioclavicular Joint. In: Rockwood CA jr, Green DP, eds. Fractures in Adults, Vol. 1. Philadelphia: Lippincott-Raven; 1984

[13] Jäger M, Breitner S. Therapiebezogene Klassifikation der lateralen Klavikulafraktur. Unfallheilkunde 1984; 87: 467-473

[14] Linhart W, von Laer L. Allgemeine Gesichtspunkte zur kindorientierten Behandlung von Verletzungen. Orthopäde 2005; 34: 1169-1186

[15] Ackermann O, Eckert K. Fraktursonographie in der Notaufnahme - Indikationen und Grenzen. Notfall Rettungsmedizin 2015; 18: 483-491

[16] Wilfinger C, Höllwarth M. Laterale Klavikulafrakturen bei Kindern und Jugendlichen. Unfallchirurg 2002; 105: 602-605

Bibliografie

DOI https://doi.org/10.1055/a-0623-6137

OP-JOURNAL 2018; 34: 270-278 @ Georg Thieme Verlag KC Stuttgart · New York ISSN 0178-1715 